\title{
THE SURGERY OF CARDIOSPASM
}

\author{
By Rodney Maingot, F.R.C.S.Eng. \\ Surgeon, Royal Free Hospital London, and Southend General Hospital
}

Cardiospasm is a condition in which there is a dilatation, hypertrophy and lengthening of the oesophagus, associated with non-organic obstruction of the cardia or, to be more precise, of the lower 3 to $6 \mathrm{~cm}$. of the oesophagus.

As the cause of the disease is as yet unknown, the synonym-idiopathic dilatation of the oesophagus - as used by Sweet (1947) and others, is appropriate.

Einhorn (1888) accurately described a case of cardiospasm and suggested that the obstruction was due to a lack of the reflex relaxation or opening of the cardia during the act of swallowing, whilst Hurst (I9I3) propounded the same theory and coined the term ' achalasia' which means absence of relaxation.

Rake (1926) considered that the condition was due to progressive degeneration of Auerbach's plexus in the region of the epicardia. Wooler (1948), however, believes, as I do myself, that the ganglionic degeneration is secondary to dilatation, inflammation and fibrosis of the oesophagus, and is not the cause of cardiospasm.

We must admit that we do not know the cause of this interesting disease, but it would appear to me that the disordered neuromuscular mechanism affecting the epicardia is a condition of spasm rather than of inco-ordination of the peristaltic wave.

Cardiospasm accounts for some 20 per cent. of cases of dysphagia.

The condition occurs twice as commonly in females as in males, and no age is exempt.

Whilst cardiospasm is not infrequently observed in infancy and childhood, most patients present themselves for treatment during the third or fourth decade. The fact that many cases are observed during the early years of life and that many of the sufferers from this disease give a long history of dysphagia dating back to early childhood, would lend a measure of support to the congenital theory (Walton, 1925).

In adults; however; the onset often coincides with some type of psychological trauma. The cardia and lower reaches of the oesophagus in these highly-strung patients appear to be con- trolled by their emotions, and tightly contract when they are depressed, excited or angry.

Successful advice and treatment by a psychiatrist or a family doctor should be advocated for these early mild cases in which underlying structural changes are so frequently shown to be minimal.

In frankly established cases, psychological or palliative treatment will be of no avail, and cure or amelioration of the distressing symptoms can only be achieved by the disruption of the obstructive mechanism in the hiatal oesophagus by means of a hydrostatic bag or by a well-planned surgical attack.

\section{Pathological Features}

The characteristic pathological features are dilatation, hypertrophy and lengthening of theo oesophagus. At operation the following charac-? teristics are noted in every case : the cardiac orifice itself is normal in appearance and diameter, while just above the cardia the terminal 3 to $6 \mathrm{~cm}$. of the oesophagus are thin, atrophic and narrowed, often having a diameter of not more than $x$ to $\mathrm{I} .5$ $\mathrm{cm}$.

There are no signs of any adhesions or abnormal congenital bands, and there are no evidences of inflammation past or present or of extrinsic constricting agents in the region of the cardia or of the lower end of the oesophagus.

Above this narrowed segment the thick-walled, bloated, funnel-shaped oesophagus can be seen disappearing upwards into the chest.

The dilatation of the oesophagus varies in shape according to the stage of the disease.

Thus, in a recent case it is fusiform, later on it becomes flask-shaped, while the sigmoid or Jshaped varieties represent an advanced stage.

Usually the dilatation is most obvious in the lower half, but in long-standing cases it may extend as far upwards as the neck and even involve the pharynx.

As the disease progresses, the enomously distended oesophagus swings to the right in the chest, pursuing a serpentine course, and may, by virtue of its retained, semi-solid, decomposing contents, compress the heart, aorta or trachea. 
Certain complications may arise : haemorrhage from the ulcerated areas in the mucosa, acute perforation, inhalation pneumonia, bronchiectasis, lung abscess, avitaminosis, and arthritis which is probably due to toxic absorption.

\section{Diagnosis}

The cardinal clinical features of cardiospasm are dysphagia, regurgitation and retrosternal pain.

The disease usually pursues a leisurely but nevertheless relentless course, although in some instances there may be some brief periods of respite.

Patients suffering from cardiospasm have been described by Wooler as being 'oesophagusconscious.'

They can actually feel the gullet go into intense spasm if they become excited, and they are conscious of its relaxing on taking a hot drink.

The first few mouthfuls of food may cause little discomfort, but further swallowing produces the sensation that the food is sticking behind the lower half of the breast bone.

If the patients continue to eat rapidly, food gushes up into the pharynx and this may produce a fit of coughing.
The symptoms may, of course, vary from day to day, but in a general way they gradually become worse as the disease inevitably progresses.

During a late stage in the disorder, dysphagia and sternal discomfort after eating increase in severity.

These patients often try to combat the symptoms by eating slowly, selecting their diet with the utmost circumspection, and by washing down solids with fluid drinks. But they lose weight rapidly because they cannot swallow sufficient food.

If the inferior constrictor of the pharynx is not working properly, they suffer from regurgitation of food into the mouth at night, especially when in bed. They become thin and anaemic and may. eventually present a severe degree of inanition.

$A$ barium meal $X$-ray examination gives a distinct picture of cardiospasm (Fig. I).

The blockage at the lower end of the gullet is either complete or incomplete.

Early on there is the intermittent passage of small quantities of barium into the stomach.

The fusiform oesophagus is lashed into vigorous writhing activity in a brave and determined attempt to force the opaque medium through the ribbon-like channel into the capacious stomach.

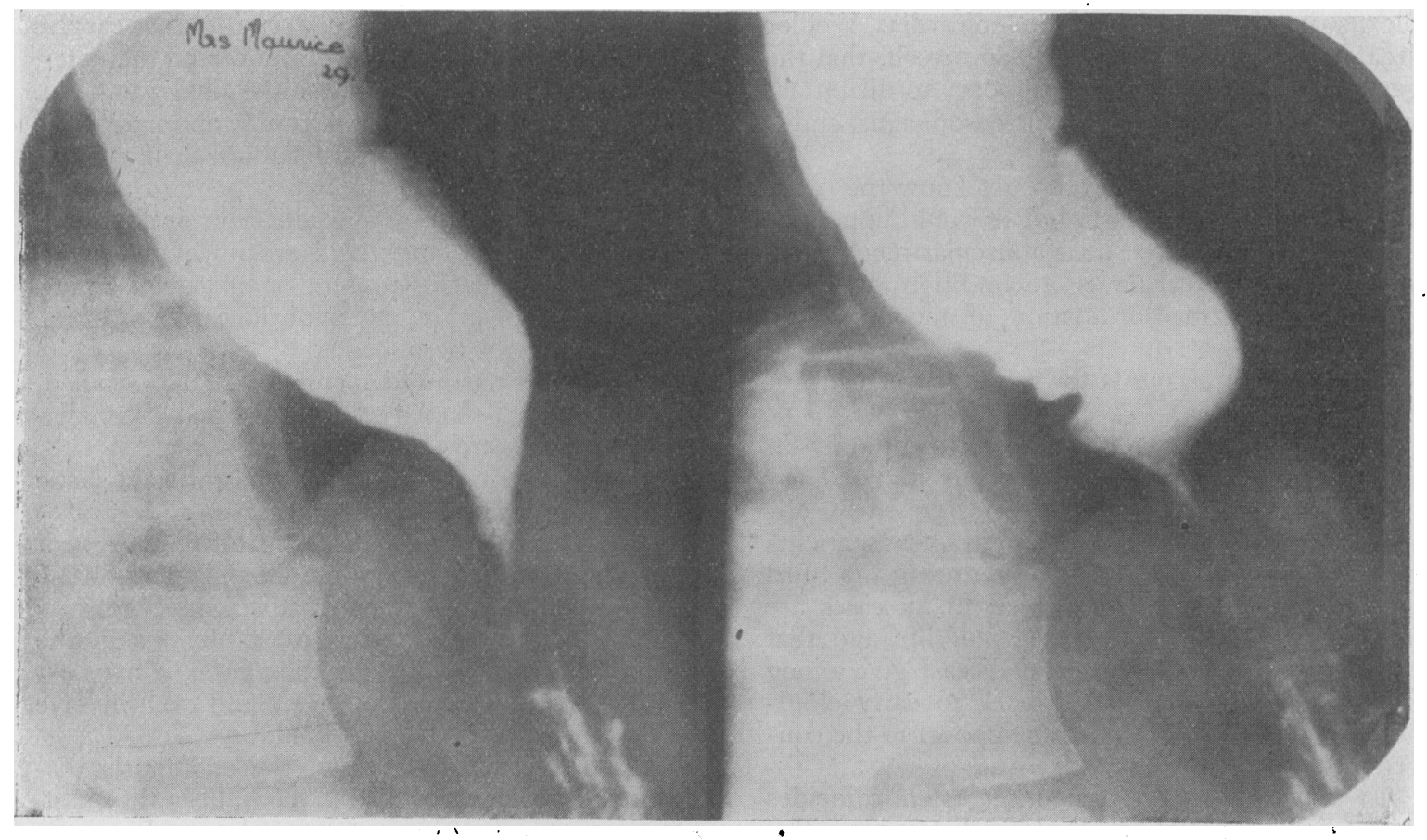

FIG. 1.-Typical X-ray appearances in a case of cardiospasm. 
In the last phase there is obstruction and marked dilatation with sagging, little or no barium entering the stomach.

The S- or J-shaped gullet is now a huge, elongated, patulous sac, incapable of vigorous peristaltic movement, although the characteristic worm-like movements of the circular coat may be seen on screening, indicating that the end of the struggle is in sight.

In a well-marked case, the lower end of the oesophagus will be seen to be narrowed, and ends in a smooth, rounded, symmetrical cone. The tip of the oesophagus is outlined as a nipple-like projection, the tip pointing to the left below the diaphragm.

In the differential diagnosis reflex spasm, benign stricture, peptic ulceration, so-called congenital stenosis and cancer must be borne in mind.

\section{Treatment}

The treatment of cardiospasm may be outlined as follows :-

(a) Medical. (I) Bland nutritious non-residue diet. (2) Skilful advice and suggestion by psychiatrist or family physician. (3) Antispasmodic drugs, such as amylnitrite or octylnitrite.

(b) Dilatations. (I) Hurst's graduated mercuryfilled bougies. (2) Plummer's hydrostatic bag. (3) Negus's modification of Tucker's bag.

\section{(c) Operation.}

Medical management, psychiatry and the use of antispasmodics, and more particularly inhalations of octylnitrite, may temporarily abolish the obstruction and afford some measure of relief.

The old-fashioned method of dilatation with Hurst's mercury tubes or oesophageal bougies has been completely abandoned, as it is most distressing to the patient and rarely leads to a permanent cure.

Today the treatment of an established case of cardiospasm is by means of rupture of the constricting circular muscular fibres of the eipicardia by means of Negus's modification of Tucker's bag, or else by operation.

Negus's* bag, when skilfully employed, will cure 70 per cent. of the patients, and there will be improvement in a further 20 per cent. In 10 per cent. it is impossible or dangerous to employ the hydrostatic bag, and operation to overcome the obstruction is clearly required.

* There are two sizes of Negus's dilators for adults : the three-fingers' breadth which holds $40 \mathrm{cc}$. of water, and the four-fingers' breadth which holds $60 \mathrm{cc}$. of water. The three-fingers' breadth dilator is used for the first dilatation in an adult, and the four-fingers' bread $h$ for subsequent dilatations.

\section{Indications for Operation}

(I) When, owing to the great size and sagging of the flask, dilatation under direct vision is a hazardous undertaking or is not feasible.

(2) When the patient has failed to respond to one or more courses of treatment with the hydrostatic dilator.

(3) In infancy and childhood. Young patients frequently fail to benefit by dilatation methods, and division of the spastic band of muscles is strikingly successful.

(4) In all those cases where the diagnosis is in doubt. Here surgical enquiry is imperative in order to rule out the possibility of cancer of the cardia or of the lower end of the oesophagus.

\section{Operations for Cardiospasm}

(r) Mikulicz. Transgastric digital dilatation of the cardiac sphincter and lower end of the oesophagus.

(2) Oesophagogastrostomy. Anastomosis of the oesophagus to the stomach (after the Finney type of gastroduodenostomy).

(3) Oesophagoplasty. Longitudinal division of all the coats of the cardia and lower end of the oesophagus, followed by suture of the transverse axis (after the Heineke-Mikulicz principle for pyloric obstruction).

(4) Extramucous oesophagogardiomyotomy. Heller's operation (based on Rammstedt's operation for infantile pyloric stenosis).

The Mikulicz (1882) transgastric blind digital stretching of the cardiac sphincter and lower end of the oesophagus is an unscientific operation and should not, in my opinion, be practised. The main danger here is rupture of the lower end of the oesophagus followed by mediastinitis. Also the digital dilatation fails to relieve the obstruction in over 25 per cent. of the cases thus treated.

Oesophagogastrostomy is of two types, Heyrovsky's (1912), which is a side-to-side anastomosis between the oesophagus and the cardia; or Grondahl's (I916), employing the Finney type of operation for duodenal scar stenosis.

The Finney type of repair is more popular and has the weighty support of Ochsner and DeBakey (1940), Gray (1941), Clagett (1948), Womack (1938), Garlock (1947) and most British surgeons.

The essential steps in the technique of Grondahl's oesophagogastrostomy will be appreciated by referring to Fig. 2.

This operation, however, has two serious drawbacks ; one is marked regurgitation of food into the gullet when the patient is reclining, and the other is the possible onset of peptic ulceration in the lowest segment of the oesophagus from the action of the gastric chyme on the sensitive oesophageal mucosa. 


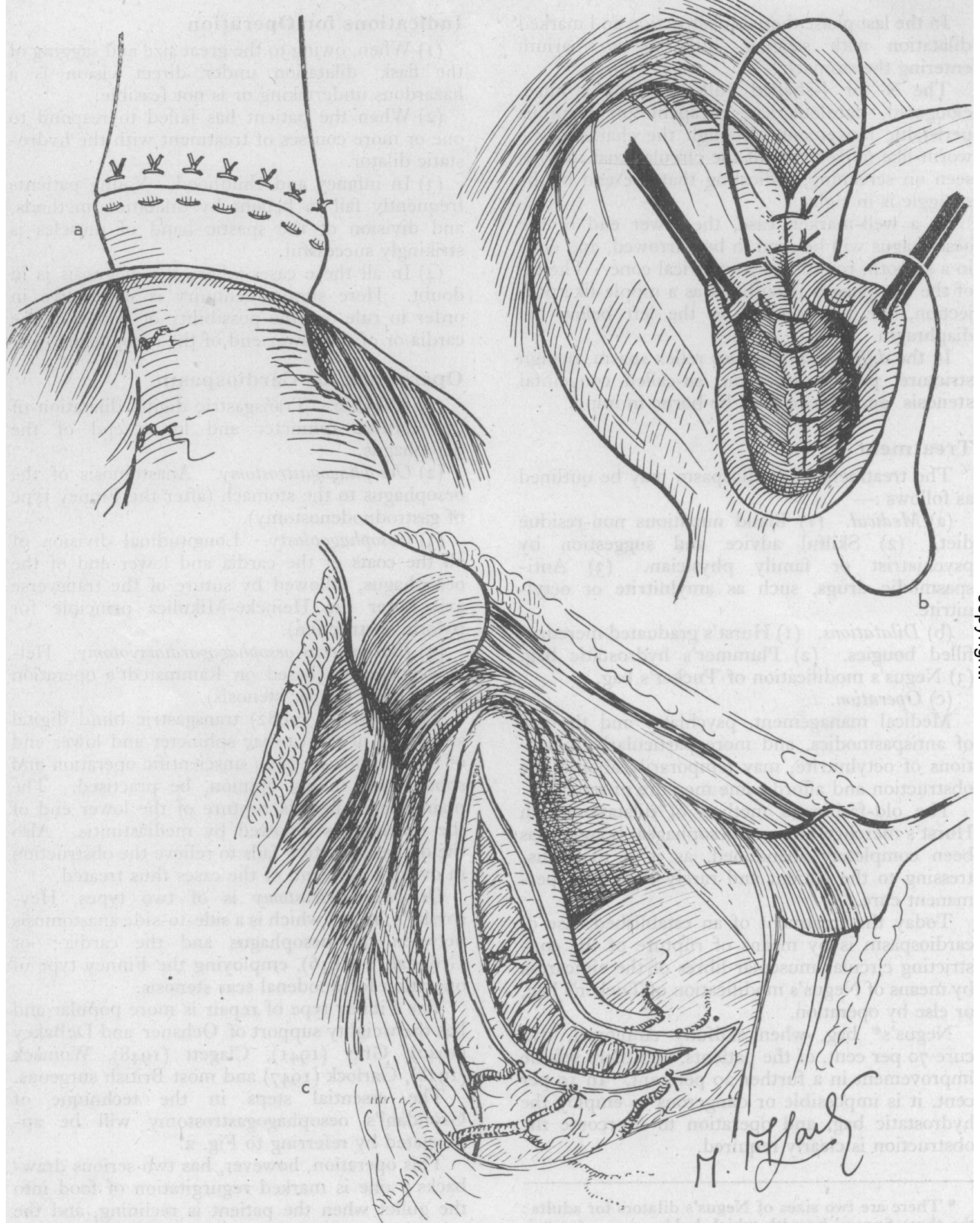

FIG. 2.-Operations for cardiospasm. (a) Oesophagocardioplasty." This operation is performed through the chest. The longitudinal incision, which was made through the flask-shaped oesophagus, epicardia, cardia and stomach, is sutured in the transverse axis. When the suturing is completed a wide communication is effected between the oesophagus and the stomach. The suture line will be seen to lie above the diaphragm. (b) Oesophagogastrostomy. This operation is based on Finney's gastroduodenostomy. (c) Heller's Operation completed. Note the length of the incision which is made through the longitudinal and circular coats of the lower end of the oesophagus and cardiac region. 
Oesophagocardioplasty is the longitudinal division of all coats of the cardia and adjacent stomach and lower end of the oesophagus, including at least $5 \mathrm{~cm}$. of the dilated flask beyond the epicardia, followed by suture in the transverse axis after the Heineke-Mikulicz principle (Fig. 3). This operation has of necessity to be performed through the chest ; it is difficult to execute, suturing is somewhat complicated, and it is often followed by minor chest complications and intercostal ache. It has been warmly advocated by Sweet (I947), who presented a series of 14 consecutive, highly successful cases, and in England has been sponsored by Grey Turner and many thoracic surgeons.

None of these operations, however, can be compared with Heller's extramucous oesophagocardiomyotomy, which is performed through an epigastric incision with speed, safety and precision.

The immediate and late results are excellent in every respect, and it is a simple procedure which can be taught to any abdominal surgeon to whom meticulous and precise work makes a special appeal.

It is associated with a negligible mortality, and a stay in hospital which does not in the average case exceed eight days. There is no regurgitation of gastric contents into the oesophagus or mouth, and there are no teasing complications such as peptic ulceration. The patient can furthermore enjoy hearty meals without the slightest restraint or discomfort.

In a personal series of 39 cases cure was obtained in 38 , there being one operative death from atelectasis in a feeble, emaciated patient aged 75 , who had suffered from dysphagia for about $5^{\circ}$ years.

\section{Results of Operations for Cardiospasm} (Personal Series)

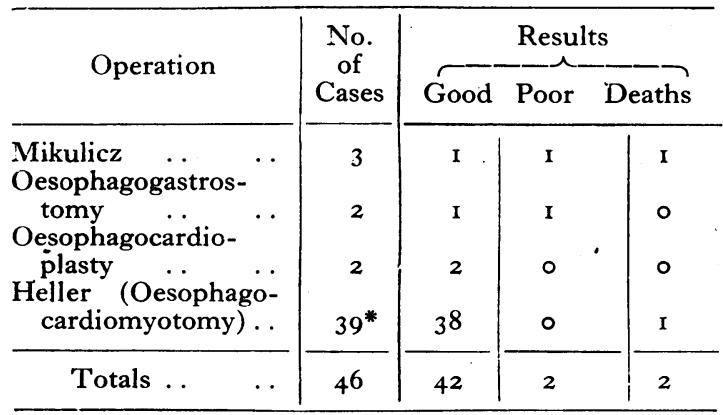

* In this series are included eight cases reported in the October, 1944, issue of the Post Graduate Medical Fournal.

\section{The Technique of Extramucous Oeso- phagocardiomyotomy}

Heller's operation was first described in 1913 and again in 1921 . He reported 16 cases operated on by him without fatality and with good results in 12.

The operation was first suggested by Gottstein in rgor, but Heller when he performed his series. of operations made two longitudinal incisions, an anterior and a posterior, to ensure thorough. division of all the constricting circular muscle fibres.

The modern operation is based on Zaaiger's. procedure (1923), as this surgeon considered that an anteriorly-placed incision through the coats of the oesophagus, epicardia and cardia itself down to the mucous membrane was all that was needed. to produce a cure.

We name cerțain operations after certain well-. known surgeons merely because it is customary and more convenient ; but it is often the best known - sponsor rather than the originator of a particular operation who receives all the praise and credit.

In the pre-operative preparation, lavage of the oesophagus is carried out once or twice daily for a few days, using a weak solution of sodium bicarbonate, until all signs of oesophagitis have disappeared.

The patient is given a well-balanced fluid diet rich in vitamins, but no solids are allowed.

A few hours before operation is due to commence, the oesophagus is thoroughly irrigated with warm normal saline solution, after which a Ryle tube is passed through the nostril into the stomach and the gastric contents are aspirated.

The Ryle tube is allowed to remain in situ during the operation, as this greatly facilitates the procedure, and also for the first one or two postoperative days.

The abdomen is opened through a lengthy left epigastric paramedian incision, and the stomach is drawn firmly downwards, and the left lateral hepatic ligament divided, as suggested by Lambert (I9I3), in order to permit of ready retraction of the left lobe of the liver and adequate exposure of the cardia.

The peritoneum over the oesophagus is incised for $5 \mathrm{~cm}$. or so at the site of its reflection on to the diaphragm, and by blunt and sharp dissection the oesophagus is liberated circumferentially.

When the oesophagus has been fully freed from the hiatus, a sling of rubber tubing is placed around the gullet to permit of traction downwards on the oesophagus. This traction on the sling aids in the mobilization of the oesophagus which is gradually pulled downwards into the peritoneal cavity for 5 to $12 \mathrm{~cm}$.

A longitudinal incision, 10 to $15 \mathrm{~cm}$. in length, is made through the muscular coats of the flaskshaped oesophagus, through the spastic zone and cardiac region and curving slightly upwards towards the fundus and down to the mucosa. 
The incision is placed anteriorly and is made very cautiously through the longitudinal muscle coat and then through the thin, somewhat adherent circular coat, dividing all the fibres until the oesophageal mucosa and the gastric mucosa bulge boldly outwards without restraint (Fig. 4).

Some small blood vessels lying on the mucosa may need ligation with fine silk.

In the average case, as I have said, the incision is fully ro to $15 \mathrm{~cm}$. long, and the exposed mucosa being translucent, the Ryle tube and bubbles of gas in the gastric fluid can be seen through the diaphanous sheet of bowel.

The operation is completed by grasping the body of the stomach and squeezing it to force air and gastric juice upwards through the oesophagus to make sure that the thin exposed mucous membrane of the oesophagus, cardia and stomach itself is not punctured.

If the mucous membrane is accidentally nicked or torn, and in nine of my cases this was so, the hole can be securely closed with a series of fine silk stitches without fear of subsequent leakage.

It is important to draw the left vagus nerve out of harm's way after the mobilization of the oesophagus has been completed and before the longitudinal incision has been commenced.

The operation is based on the principle of Rammstedt's pyloromyotomy for infantile pyloric stenosis.

It differs from this procedure in that there is no gritty tumour to incise and there is no obstructing band or strictured area which can be seen with the naked eye.

Many observers have stated that the dilated oesophagus following this operation, although functioning normally, does not contract down to its former size. While this may be so for the advanced and neglected types of cardiospasm, it is certainly not true of the average case, as my numerous post-operative skiagrams will clearly demonstrate. Therefore, the more advanced the case, the smaller is the subsequent contraction which obtains.

In the post-operative phase no special treatment is required apart from keeping the patient on a fluid diet for the first three or four days. After this, semi-solid nourishment is allowed, and before the patient is discharged on the seventh or eighth day he is given full diet without restriction.

\section{Conclusions}

(I) Fully 70 per cent. of cases of cardiospasm can be cured by the hydrostatic bag, and about 20 per cent. are greatly relieved.

(2) Operation is called for in the remaining ro per cent. of cases, and in a percentage of those who have to be subjected to repeated dilatations.

(3) The main indications for operation are :-

(a) Failure of the dilatation method.

(b) The dilatation method is impracticable.

(c) The impossibility in some cases of ruling out carcinoma ; and

(d) When the disease is encountered in infancy and childhood.

(4) There are three good operations for cardiospasm :-

(a) Oesophagogastrostomy.

(b) Oesophagocardioplasty.

(c) Heller's operation.

(5) The operation of choice, in my opinion, Heller's or the slight modification which I have here presented.

\section{BIBLIOGRAPHY}

EINHORN, M. (1888), Med. Rec., 37, 75 I. GRAY, H. K. (1941), $\mathfrak{f}$. Thorac. Surg., 10, 220. GRONDAHL, N. B. (1916), Nor. klin. for forhand, II, 236. HELLER, E. (1913), Mitt. Grenzgeh. Med. Chir., 57, 14 I. HELLER, E. (1921), Verhardl. dtsch. Gesellsch. Chir., 45, 144. HURST, A. (1913), Proc. R. Soc. Med., 7, 150.

MAINGOT, R. (1944), Post Grad. Med. F., $20,278$.

MAINGOT, R. (1948), 'Abdominal Operations,' and edition Appleton-Century-Crofts, New York.

NEGUS, V. E. (1948), Personal communication.

OCHSNER, A., and DE BAKEY, M. (1940), Arch. Surg., 4I, I146.

RAKE, G. W. (1926), Guy's Hosp. Rep., 76, 145.

SWEET, R. H. (1947), Surg. Clin. N. Am., Oct., p. I 28.

WALTON, J. (1925), Brit. F. Surg., 12, 701.

WOOLER, G. H. (1948), Thorax, 3, 53.

WORMACK, N. A. (1938), Surg. Clin. N. Am., Oct., p. 1241

ZAAIGER, J. H. (1923), Ann. Surg.. 77, 615 